\title{
Why is the Red Rectangle Unique?
}

\author{
H. Van Winckel \\ Instituut voor Sterrenkunde, KU Leuven, Celestijnenlaan 200D, 3001 Leuven, Belgium \\ email: Hans.VanWinckel@ster .kuleuven. be
}

\begin{abstract}
The Red Rectangle is a spectacular bipolar nebula centred around the eccentric evolved binary HD 44179. The nebula is rich in spectral signatures: it is the brightest source of the Extended Red Emission (ERE), of the Blue Luminescence (BL) as well as one of the brightest sources of mid-IR PAH bands. Moreover, and unique to this source, a plethora of narrow emission lines is detected on top of the diffuse ERE. Some of these molecular features show rotational contours which become narrower at larger angular separation from the central engine. Some converge to wavelengths and profiles of narrow DIBs. All these observations show that the Red Rectangle is a very rich laboratory to study active astrochemical processes within the nebula. We also address, but fail to answer, the question why this source is so unique among the many observed post-AGB binary analogues.
\end{abstract}

Keywords. stars: individual (HD 44179), stars: AGB and post-AGB, ISM: molecules, astrochemistry, (stars:) binaries: spectroscopic, (stars:) circumstellar matter, (ISM:) reflection nebulae

\section{The Red Rectangle Nebula}

The Red Rectangle (see Figure 1, Cohen et al. 2004) is a spectacular bipolar nebula surrounding the central star HD 44179. Figure 1 displays the inner structure only, as deep coronographic images show that the nebular emission can be traced to about one arcminute on either side of the centre. These HST images show that the centre is resolved and a dark lane of optically thick dust obscures the central object from direct light. We only see scattered light in the visible to near-IR (Men'shchikov et al. 2002, Gledhill et al. 2009). The nebula is projected nearly edge-on onto the sky.

The object is famous, not only for its geometry, but also for its spectral properties. It is the brightest source of Extended Red Emission (ERE) (see Witt, these proceedings), one of the brightest sources of the mid-IR PAH bands (see Peeters, these proceedings) and displays Blue Luminescence (BL) emission (Vijh et al. 2004, Witt these proceedings).

In this contribution we focus first on the DIB connection (Section 2). Contrary to the ISM, the active chemistry in the Red Rectangle nebula occurs under rather well constrained conditions in a more homogeneous chemical environment. We therefore focus on these conditions by giving an overview of the properties of the central object in Section 3 and display the system as an evolved interacting binary surrounded by a stable dusty circumbinary disc. In the next section, we argue that these type of binaries are commonly detected, and this both in the Galaxy as in the Large and Small Magellanic Clouds (LMC and SMC). The natural astrophysical question which arises then is why the Red Rectangle circumstellar chemical processes are so unique and not found in other systems.

\section{The Red Rectangle - DIB connection}

The nebula is extremely rich in molecular emission, many of which are unique to this particular object. The diffuse emission is covered elsewhere in these proceedings and I 

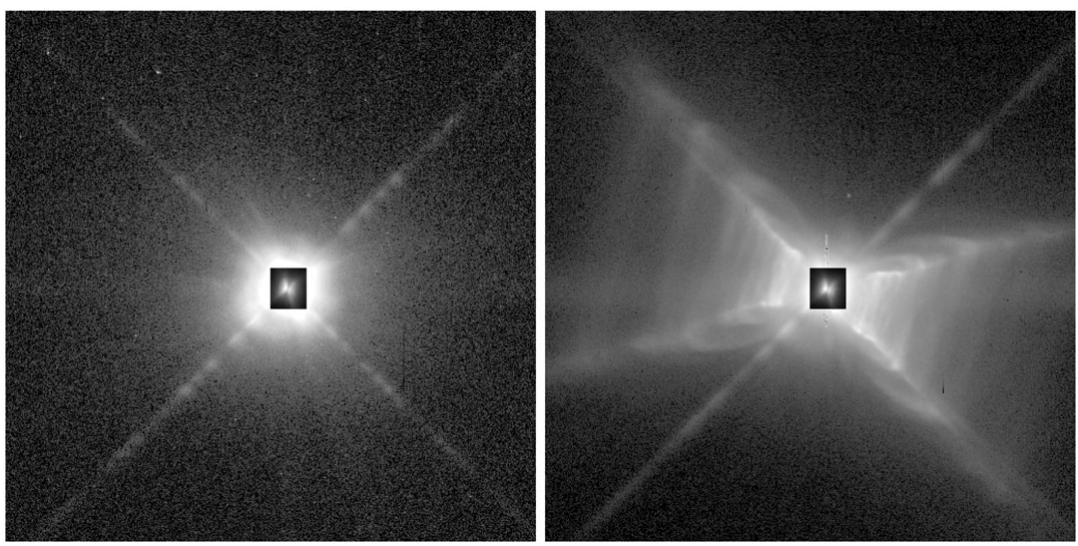

Figure 1. Illustration of the strong ERE emission in the Red Rectangle. Left is the HST image of the central region in the F467W filter, with the total field-of-view of $23.5 \operatorname{arcsec}^{2}$. This image shows scattered light only. The inner region has a different cut as to increase the total dynamic range. The dark lane is clearly resolved and we only see scattered light. On the right, the HST image is displayed in the FW622W filter, the transmission curve of which peaks in the ERE. In this filter, the nebular structures (the X-shape, the ladder rungs etc.) stand out. Outside the ERE, the Red Rectangle is a Blue Circle but within the ERE emission itself, the complex nebular structures reveal themselves (see also Cohen et al. 2004).

focus on the narrow emission features on top of the ERE. These features are illustrated in Figure 2. The features were originally found by Schmidt, Cohen \& Margon (1980) and a full overview of all detected features can be found in Van Winckel, Cohen \& Gull (2002).

The nebula is faint but as it is projected nearly edge-on onto the sky, one can add spectra taken at similar angular distance from the central object to increase signal-tonoise (e.g. Sarre et al. 1995, Glinsky et al. 2002, Wehres et al. 2011). These spatially resolved spectra show that most, but not all, narrow features show typical molecular rotational contours and a decrease of rotationally excited states at larger distances from the central engine (Figure 3). The strongest of these features converge to the narrow DIB's at $\lambda 5797.11, \lambda 5849.78$, and $\lambda 6613.72 \AA$ (Sarre et al. 2005 , Sarre these proceedings). At the limit of detectability, this convergence is not yet complete, so there remains some doubt to whether the actual carriers of these DIBs are indeed also produced in the Red Rectangle (e.g. Glinsky et al. 2002).

The spatially resolved active chemistry in the nebula is illustrated in Wheres et al. (2011) where one clearly see that around 7 arcsec from the central source, the intensity of most features is maximal. The detection of the Swann bands of the $\mathrm{C}_{2}$ radical by Wheres et al. (2010), with its very short lifetime, shows that a very active photochemistry is occurring in the nebular ejecta. Other illustrations are the detection of $\mathrm{CH}^{+}$emission, likely originating in the PDR zone very close to the star by Balm \& Jura (1992).

Apart from the Swann bands of $\mathrm{C}_{2}$, none of the narrow features on top of the ERE are identified. Moreover, they are unique to this source as, to my knowledge, they have not yet been observed elsewhere. It is therefore good to look at the central source to find indications of unique properties of the Red Rectangle.

\section{The Red Rectangle as evolved binary}

To place the Red Rectangle in a stellar evolutionary context, the properties of the central star are most indicative. The optical scattered flux is dominated by one component, 


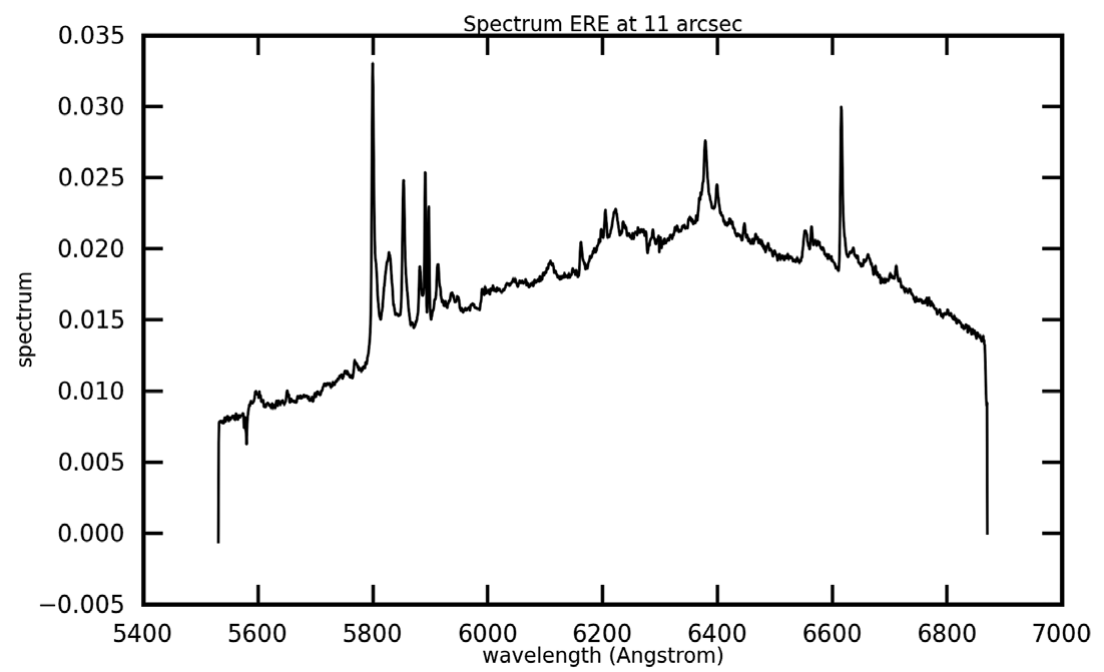

Figure 2. A spectrum of the Red-Rectangle nebula at 11 arcsec of the central object. The plethora of narrow complex emission features on top of the diffuse ERE is unique to this source. Spectrum taken from Van Winckel, Cohen \& Gull (2002).

which is an evolved luminous star, likely in a post-AGB evolutionary stage (Waelkens et al. 1996). It is a single lined spectroscopic binary with a period of 319 days and a remarkably high eccentricity of 0.38 . The semi-major axis is $\mathrm{a}_{1} \sin i=0.37 \mathrm{~A}$.U. (Van Winckel et al. 1995, slightly revised by extended time series). As we observe the object in scattered light only, the effective inclination is determined by the viewing angle of the scattering surface, which is not in the direct line-of-sight. Irrespective of the effective inclination, these orbital dimensions are too small to accommodate a full grown AGB star. The object has not evolved on single-star evolutionary tracks and must have had (or is in) a phase of strong binary interaction.

The circumbinary dust is stored in a disc which is optically thick and is seen as a dark lane in the optical images (Fig. 1). The disc is in Keplerian rotation and the kinematics were resolved by interferrometric CO observations by Bujarrabal et al. (2005). The outer regions of the gas in the disc is expanding with a very low expansion velocity of a few $\mathrm{km} / \mathrm{s}$. The Keplerian kinematics as well as the slow bipolar wind was even better resolved in the recent ALMA experiment (Bujarrabal et al. 2013). The bipolar emission is clearly resolved in the $\mathrm{J}=3-2$ and $\mathrm{J}=6-5$ rotational transition of $\mathrm{CO}$.

The mid-IR spectrum of the nebula is dominated by PAH emission. At longer wavelengths, the crystalline silicate dust emission is detected, which shows that the disc was formed before the object ejected the carbon-rich nebula (Waters et al. 1998). The longevity of the disc is also shown by the spectral index at very long wavelengths and the $\mathrm{cm}$ continuum emission can best be explained that dust processing in the disc is responsible for the creation of $\mathrm{cm}$ sized grains (Jura et al. 1997). These large grains settle efficiently in the mid-plane of the disc.

The companion is likely an unevolved main-sequence star (Waelkens et al. 1996) but as the primary is a low gravity star in a rather close orbit, the interaction is ongoing. It was demonstrated by phased resolved $\mathrm{H}_{\alpha}$ monitoring (Witt at al 2009, Thomas et al. 2013) that the primary feeds and accretion disc around the secondary, where a bipolar jet is launched with a high velocity outflow $\left(\mathrm{v}_{\max }=200 \mathrm{~km} \mathrm{~s}^{-1}\right)$ and a quite wide opening angle (half opening angle is about $35^{\circ}$ ). This accretion disc is an important ingredient in both the chemical as the geometrical properties of the nebula. 


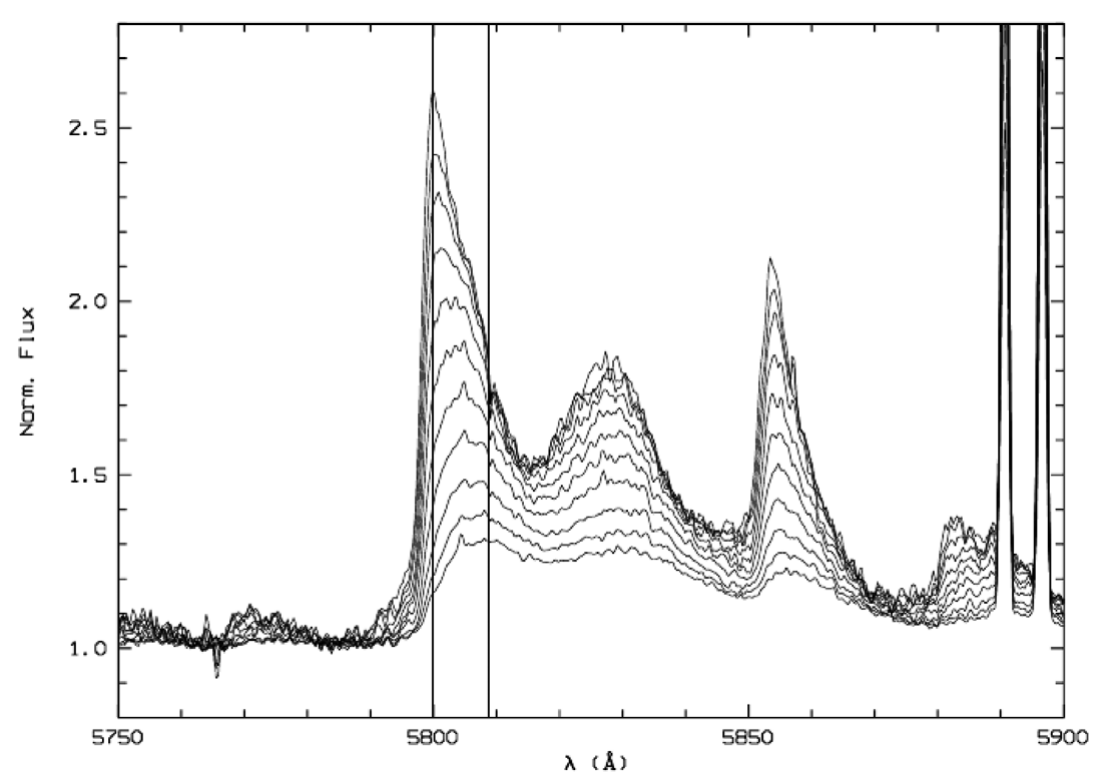

Figure 3. The rotational contours of the spectral features in the $5800 \AA$ region. The different lines trace different angular distances from the central object from 2.9 arcseconds (bottom spectrum) to 5.6 arcseconds (top spectrum). Data from Van Winckel, Cohen \& Gull (2002)

Another outstanding property of the central star is its chemical composition (Waelkens et al. 1992): the central object is a low gravity star with an effective temperature around $7500 \mathrm{~K}$. With an $\mathrm{Fe}$ abundance of $[\mathrm{Fe} / \mathrm{H}]=-3.3$ but a near solar $\mathrm{S}$ and $\mathrm{Zn}$ abundances, the photospheric chemical pattern reflects the gas-phase abundance of the interstellar medium (see also Clayton these proceedings): refractory elements, which easily condense to dust grains, are strongly deficient while non-refractory elements like S and Zn are less or not affected. HD 44179 is one of the most depleted objects we know. The depletion process itself is still poorly understood, but the basic ingredients involve a phase of dusty mass loss. The dust formation process induces the chemical fractionation as the refractory elements with a high dust-condensation temperature are preferentially locked up in solid state dust particles. A gas-dust separation process is followed by an accretion on the star of the circumstellar gas only, which results in a photosphere that is coated by a layer of circumstellar gas devoid of refractory elements. The circumstellar disc allows for a stable environment in which dust-gas separation and re-accretion can occur (Waters et al. 1992). The evolved luminous component in the Red Rectangle is strongly affected by this process. This also means that the ongoing circum-companion gas accretion will be with depleted gas devoid of the refractory elements.

\section{The Red Rectangle analogues: binary post-AGB stars}

A key property of the Red Rectangle is the Keplerian circumsystem dusty disc. In the last decades it became clear that stable discs are commonly detected around evolved objects. A distinct observational characteristic is the near-IR excess, which shows that dust, close to the central star is present, despite the fact that the objects are now not in a phase of dusty mass loss. The initial sample of de Ruyter et al. (2006) contained some 50 sources, but this is now expanded to about 80 Galactic objects (Gielen et al. 2011). Moreover, the distinct infrared properties make that also in the LMC and SMC these 


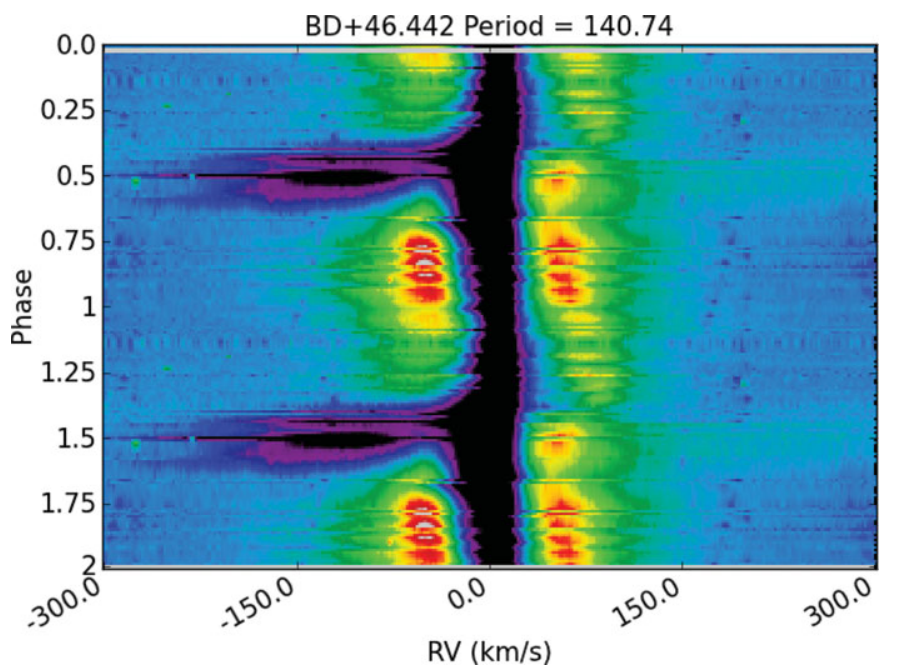

Figure 4. Time series of $H_{-} \alpha$ line profiles of BD+46.442 with an orbital period of 140.74 days. The phase of conjunction is around 0.5 when the primary is eclipsed by the jet likely originating in the circum-companion gas disc around the unevolved companion. This is similar to what is observed in the Red Rectangle (Witt et al. 2009), in a system which is seen under a different inclination. Data from Gorlova et al. (2012).

sources are being found (van Aarle et al. 2011, Kamath, Wood \& Van Winckel 2013) and they represent about half of the optically bright post-AGB candidates in these galaxies.

Subsequent radial velocity monitoring campaigns indeed proved these systems to be likely all binaries (Van Winckel et al. 2009) and the global picture that emerges is that a binary star evolved in a system which is too short to accommodate a full grown AGB star. During a badly understood phase of strong interaction, a circumbinary dusty disc was formed, but the binary system did not suffer dramatic spiral in. What we observe now is a F-G-K supergiant in a binary system, which is surrounded by a circumbinary dusty disc in a bound orbit.

It also became clear that depleted photospheres are very widespread in these systems and not limited to extreme cases like the Red Rectangle. Much milder depletion patterns are being detected (e.g. Giridhar et al. 2005, Maas et al. 2005, and references therein). The depleted objects are not limited to Galactic objects as in the LMC, depleted photospheres are now being found as well. Also there only among the objects surrounded by discs (Reyniers \& Van Winckel 2007, Gielen et al. 2009).

Another remarkable finding is that more circum-companion accretion discs are found. This is illustrated in Fig. 4 which shows time-resolved $\mathrm{H}_{\alpha}$ profiles of a binary (Gorlova et al. 2012). At conjunction, a blue absorption appears when the jet obscures the background luminous star and continuum photons are scattered outside the line-of-sight.

Given the number statistics, the range of orbital periods and given the range in quantified mass functions, the conclusion is that the formation, structure and evolution of the stable discs appear to be a key ingredient in our understanding of the late evolution of a very significant binary population!

\section{Why is the Red Rectangle so unique ?}

The observational evidence for the presence of stable dust structures around Galactic post-AGB binaries, as well as around post-AGB candidates in the LMC and SMC make 
that we can conclude that stable disc formation is a mainstream process in evolved stars. The Galactic radial velocity monitoring programmes reveal a close link between disc formation and the binary nature of the central star. Chemical studies have shown that photospheric depletion patters are also commonly observed. The evolved binaries avoided the strong spiral-in but also retained their hydrogen-rich envelope. They reveal themselves as binary post-AGB stars the system of which is surrounded by stable circumbinary dusty discs. In several objects, intensive monitoring shows that the central stars are interacting and that the companion is surrounded by a low-mass accretion disc.

HD 44179 and its surrounding Red Rectangle nebula, can be seen as an outstanding example of this group. Yet, it is contradictory that the rich and active circumstellar chemical processes seem to be unique to the Red Rectangle and it is not observed in any other object. It is as yet unclear which chemo-physical agent is crucial and unique and/or very short-lived in the Red Rectangle.

\section{References}

Balm, S. P. \& Jura, M. 1992, A\& A, 261, L25

Bujarrabal, V., Castro-Carrizo, A., Alcolea, J., \& Neri, R. 2005,A\&A, 441, 1031

Bujarrabal, V., Alcolea, J., Van Winckel, H., Santander-Garcia, M., \& Castro-Carrizo, A. 2013, 557, A104

Bujarrabal, V., Castro-Carrizo, A., Alcolea. J., Van Winckel, H., Sánchez Contreras, C., Santancer-García, M., Neri, R., \& Lucas, R. 2013, A\&6A, Letters, 557, L11

Cohen, M., Van Winckel, H., Bond, H. E., \& Gull, T. R. 2004, AJ, 127, 2362

de Ruyter, S., van Winckel, H., Maas, T., Lloyd Evans, T., Waters, L. B6. F. M., \& Dejonghe, H. 2006, $A \mathscr{E} A, 448,641$

Gielen, C., Van Winckel, H., Reyniers, M., et al. 2009, A\& $A$, 508, 1391

Gielen, C., Bouwman, J., Van Winckel, H., Lloyd Evans, T., Woods, P. M., Kemper, F., Marengo, M., Meixner, M., Sloan, G. C., \& Tielens, A. G. G. M. 2011, A\& A, 533, A99

Giridhar, S., Lambert, D. L., Reddy, B. E., Gonzalez, G., \& Yong, D. 2005, ApJ, 627, 432

Gledhill, T. M., Witt, A. N., Vijh, U. P., \& Davis, C. J. 2009, MNRAS 392, 1217

Glinski, R. J. \& Anderson, C. M. 2002, MNRAS, 332, 17

Gorlova, N., Van Winckel, H., Gielen, C., Raskin, G., Prins, S., Pessemier, W., Waelkens, C., Fremat, Y., Hensberge, H., Dumortier, L., Jorissen, A.,\& Van Eck, S. 2012, A $3 A$, 542, A26

Jura, M., Turner, J., \& Balm, S. P 1997, ApJ, 474, 741

Kamath, D. \& Wood, P. R., Van Winckel 2013, MNRAS, in press

Maas, T., Van Winckel, H., \& Lloyd Evans, T. 2005, A\& A, 429, 297

Men'shchikov, A. B., Schertl, D., Tuthill, P. G., Weigelt, G., \& Yungelson, L. R. 2002, A\&A 393,867

Reyniers, M. \& van Winckel, H. 2007, A\& A, 463, L1

Sarre, P. J., Miles, J. R., \& Scarrott, S. M. 1995, Science, 269, 647

Schmidt, G. D., Cohen, M., \& Margon, B. 1980, ApJ, 239, 133

Thomas, J, D., Witt, A. N., Aufdenberg, J. P., Bjorkman, J. E., Dahlstrom, J. A., Hobbs, L. M., \& York, D. G. 2013, MNRAS, 430, 1230

van Aarle, E., Van Winckel, H., Lloyd Evans, T., Ueta, T., Wood, P. R., \& Ginsburg, A. G. 2011, $A \& A, 530,90$

Van Winckel, H. 2003, ARA $\& A, 41,391$

Van Winckel, H., Cohen, M., \& Gull, T. R. 2002, A\&A, 390, 147

Van Winckel, H., Waelkens, C., \& Waters, L. B. F. M. 1995, A\&A, 293, 25

Van Winckel, H., Lloyd Evans, T., Briquet, M., et al. 2009, A\&A 505, 1221

Vijh, U. P., Witt, Adolf, N., \& Gordon, K. D. 2004, ApJ, 606, 65

Waelkens, C., Van Winckel, H., Trams, N. R., \& Waters, L. B. F. M. 1992, A\&A, 256, 15

Waelkens, C., Van Winckel, H., Waters, L. B. F. M., \& Bakker, E. J. 1996, A\&SA, 314, 17

Waters, L. B. F. M., Cami, J., de Jong, T., Molster, F. J. et al. 1998, Nature 391, 868 
Waters, L. B. F. M., Trams, N. R., \& Waelkens, C 1992, A\& A, 262, 37

Wehres, N., Romanzin, C., Linnartz, H., Van Winckel, H., \& Tielens, A. G. G. M. 2010, A\&A, 518, 36

Wehres, N., Linnartz, H., van Winckel, H.,\& Tielens, A. G. G. M., 2011, A $\& A$ 533, 28

Witt, A. N., Vijh, U. P., Hobbs, L. M., Aufdenberg, J. P., Thorburn, J. A., \& York, D. G. 2009, ApJ, 693, 1946 